\title{
Low temperature magnetic relaxation and quantum tunneling in nanocrystalline particles (abstract)
}

X. X. Zhang, J. M. Hernandez, E. C. Kroll, ${ }^{\text {a) }}$ R. Ziolo, ${ }^{\text {a) }}$ and J. Tejada

Department de Fisíca Fonamental, Universidad de Barcelona, Diagonal 647, 08028 Barcelona, Spain

We report measurements of the magnetic relaxation rate versus temperature for ferrofluid and magnetic-glass samples, which are formed by a modification of nanocomposite material consisting of nanocrystalline $\mathrm{CoFe}_{2} \mathrm{O}_{4}$ and polymer. ${ }^{1}$ The magnetic properties of the samples have also been studied by using SHE-SQUID at different temperatures $(1.8-300 \mathrm{~K})$ with low and high applied magnetic field $(-5 \mathrm{~T}$ to $5 \mathrm{~T})$. The magnetic relaxation in two samples show a perfect logarithmic dependence on the time, i.e., $M(t)=M\left(t_{0}\right)\left[1-S \ln \left(t / t_{0}\right)\right]$, in accordance with the ZFC-FC results which indicate that there is wide energy distribution. The temperature independence of magnetic viscosity $S \equiv\left[1 / M\left(t_{0}\right) d M / d\right] \ln t$ below several Kelvin for the two samples gives clear evidence of macroscopic quantum tunneling of magnetization, in accordance with current theories of quantum tunneling of magnetization. (C) 1996 American Institute of Physics. [S0021-8979(96)70108-6]

\footnotetext{
a) Also with Xerox Webster Research Center, Xerox Corporation, 800 Philips Rd. 0114-39D, Webster, NY 14580.

${ }^{1}$ R. F. Ziolo et al., Science 257, 219 (1992).
} 